\title{
Crystal Structures of Transhydrogenase Domain I with and without Bound NADH ${ }^{\dagger, \$}$
}

\author{
G. Sridhar Prasad, ${ }^{\S, \|}$ Marten Wahlberg, ${ }^{\S, \perp}$ Vandana Sridhar,,,$\|$ Vidyasankar Sundaresan,,$^{\S}$ Mutsuo Yamaguchi,, \\ Youssef Hatefi," and C. David Stout*,\$ \\ Division of Biochemistry, Department of Molecular and Experimental Medicine, and Department of Molecular Biology, \\ The Scripps Research Institute, La Jolla, California 92037-1093
}

Received March 29, 2002; Revised Manuscript Received August 12, 2002

\begin{abstract}
Transhydrogenase $(\mathrm{TH})$ is a dimeric integral membrane enzyme in mitochondria and prokaryotes that couples proton translocation across a membrane with hydride transfer between $\mathrm{NAD}(\mathrm{H})$ and NADP(H) in soluble domains. Crystal structures of the $\mathrm{NAD}(\mathrm{H})$ binding $\alpha 1$ subunit (domain I) of Rhodospirillum rubrum $\mathrm{TH}$ have been determined at $1.8 \AA$ resolution in the absence of dinucleotide and at $1.9 \AA$ resolution with NADH bound. Each structure contains two domain I dimers in the asymmetric unit (AB and $\mathrm{CD}$ ); the dimers are intimately associated and related by noncrystallographic 2-fold axes. NADH binds to subunits $\mathrm{A}$ and $\mathrm{D}$, consistent with the half-of-the-sites reactivity of the enzyme. The conformation of NADH in subunits A and D is very similar; the nicotinamide is in the anti conformation, the A-face is exposed to solvent, and both N7 and O7 participate in hydrogen bonds. Comparison of subunits A and D to six independent copies of the subunit without bound NADH reveals multiple conformations for residues and loops surrounding the NADH site, indicating flexibility for binding and release of the substrate (product). The NADH-bound structure is also compared to the structures of $R$. rubrum domain I with NAD bound (PDB code 1F8G) and with NAD bound in complex with domain III of TH (PDB code 1HZZ). The NADH- vs NAD-bound domain I structures reveal conformational differences in conserved residues in the $\mathrm{NAD}(\mathrm{H})$ binding site and in dinucleotide conformation that are correlated with the net charge, i.e., oxidation state, of the nicotinamides. The comparisons illustrate how nicotinamide oxidation state can affect the domain I conformation, which is relevant to the hydride transfer step of the overall reaction.
\end{abstract}

The energy-transducing nicotinamide nucleotide transhydrogenases of eukaryotic mitochondria and bacteria are homodimeric integral membrane proteins of monomer molecular mass of about $110 \mathrm{kDa}$. They catalyze the direct and stereospecific transfer of a hydride ion between the $4 \mathrm{~A}$ position of $\operatorname{NAD}(\mathrm{H})^{1}$ and the $4 \mathrm{~B}$ position of $\mathrm{NADP}(\mathrm{H})$ in a reaction that is coupled to transmembrane proton translocation with a $\mathrm{H}^{+} / \mathrm{H}^{-}$stoichiometry of $n=1$ (eq 1) (1-4).

$\mathrm{NADH}+\mathrm{NADP}+n \mathrm{H}^{+}{ }_{\text {out }} \rightleftharpoons \mathrm{NAD}+\mathrm{NADPH}+n \mathrm{H}^{+}{ }_{\text {in }}$

In bovine submitochondrial particles, the proton motive force (pmf) accelerates the forward reaction 10-12-fold, and shifts

\footnotetext{
† This research was supported by NIH Grant GM61545 to Y.H. and C.D.S.

$¥$ PDB ID codes: 1L7D and 1L7E.

* Corresponding author. Telephone: 858-784-8738. Fax: 858-7842857. E-mail: dave@ scripps.edu.

${ }^{\S}$ Department of Molecular Biology, The Scripps Research Institute.

"Present address: Syrrx, Inc., 10450 Science Center Dr., San Diego, CA 92121.

${ }^{\perp}$ Present address: Institute for Cell and Molecular Biology, Uppsala University, S-751 24 Uppsala, Sweden.

\# Division of Biochemistry, Department of Molecular and Experimental Medicine, The Scripps Research Institute.

${ }^{1}$ Abbreviations: NAD, nicotinamide adenine dinucleotide; NADH, dihydronicotinamide adenine dinucleotide; SAS, single wavelength anomalous scattering; MAD, multiple wavelength anomalous dispersion; FSBA, $p$-fluorosulfonylbenzoyl-5'-adenosine; DCCD, $N, N^{\prime}$-dicyclohexylcarbodiimide; EEDQ, $N$-(ethoxycarbonyl)-2-ethoxy-1,2-dihydroquinoline.
}

the equilibrium toward product formation. In the reverse direction, transhydrogenation from NADPH to NAD results in outward proton translocation and creation of a pmf. Because there is essentially no difference in the reduction potential of the nicotinamide cofactors, the driving force for proton translocation coupled to the reverse reaction is the difference in binding affinities for substrates (NADPH, NAD) and products (NADH, NADP). In mammalian mitochondria, a function of TH is to produce NADPH for reduction of toxic $\mathrm{H}_{2} \mathrm{O}_{2}$ by glutathione reductase and glutathione peroxidase.

TH monomers are composed of three domains: a 400430-residue hydrophilic domain I, a 360-400-residue hydrophobic domain II, and a 200-residue hydrophilic domain III. In mammalian $\mathrm{TH}$, the domains are arranged in a single polypeptide as domains I-II-III; in bacterial TH, the domains are distributed into two or three subunits $(1,2,5)$. Domains I and III are extramembranous while domain II is membraneintercalated and contains the proton channel. The binding sites for $\mathrm{NAD}(\mathrm{H})$ and $\mathrm{NADP}(\mathrm{H})$ reside separately in domains I and III, respectively $(1,2,5)$; hydride exchange between domains I and III is coupled to proton transfer involving domain II (eq 1). In Rhodospirillum rubrum, TH is comprised of three separate polypeptides, $\alpha 1, \alpha 2$, and $\beta(1,6)$ : the $\alpha 1$ subunit corresponds to domain I; the $\alpha 2$ subunit contains a short C-terminal segment of domain I and the $\mathrm{N}$-terminal portion of domain II; the $\beta$ subunit contains the remainder of domain II and all of domain III.

To date, structural studies of TH have focused on recombinant preparations of the hydrophilic nucleotide 
binding domains I and III. Crystal structures of bovine and human domain III with NADP bound have been determined $(7,8)$. This $20 \mathrm{kDa}$ domain contains a classical nucleotide binding fold, but NADP is bound in an inverted orientation from that normally observed for this fold, exposing the nicotinamide ring for direct contact with $\mathrm{NAD}(\mathrm{H})$ in domain I. A crystal structure of Rhodospirillum rubrum $\mathrm{TH}$ domain I ( $\alpha 1$ subunit) with NAD bound containing two dimers of $40 \mathrm{kDa}$ subunits has been determined (9; PDB code 1F8G). In this structure, the domain I subunit is comprised of two subdomains, dI.1 and dI.2, each with a Rossmann nucleotide binding fold, arranged about a deep cleft. The NAD binding site resides within this cleft at the C-terminal edge of the parallel $\beta$-sheet in the dI.2 subdomain. The domain I dimer contains a local 2-fold axis of pseudosymmetry such that the two NAD binding sites face away from each other. Both the dI.1 and dI.2 subdomains are involved in formation of the dimer, and in the crystal structure of the NAD complex, each dimer in the asymmetric unit contains one well-ordered and one disordered NAD (9).

A structure of a cocrystallized complex between $R$. rubrum domains I and III has been determined revealing details of the interactions in the domain I:domain III interface (10; PDB code $1 \mathrm{HZZ}$ ). In this complex, the $\mathrm{TH}$ domain I dimer and domain III monomer are very similar to the structures observed in crystals of these individual TH components (79 ). The cocrystal structure reveals a close association of a single domain III monomer with the domain I dimer; the interaction involves conserved residues in loops of domain III surrounding NADP, present in the crystals, with conserved residues in domain I from subdomains dI.1 and dI.2 in the ' $\mathrm{B}$ ' subunit, and from subdomain dI.2 in the 'A' subunit (10). Hence, the crystallized complex is a 'heterotrimer' containing a dimer of domain I subunits in association with one copy of domain III, even though intact, membrane-bound $\mathrm{TH}$ is expected to be a homodimer. The structure suggests that interaction of domain III with domain I subunits is mutually exclusive, such that only one domain III subunit interacts at a time (10), in the 'occluded' form, as observed, and in an 'open' form, corresponding to the other domain III subunit in the holoenzyme. This feature of the complex is incorporated into an 'alternating site, binding change mechanism' for proton translocation by $\mathrm{TH}(11)$. The direction of the 2 -fold axis in the heterotrimeric complex further implies that domain III resides between domain I (in the cytosol) and domain II, which is intercalated in the membrane (11).

The heterotrimeric domain I:domain III complex contains well-ordered NAD bound in the 'A' copy of domain I, but disordered NAD in the ' $\mathrm{B}$ ' copy, which is the subunit that interacts closely with domain III (10). To visualize the juxtaposition of NAD and NADP in the complex, NAD was modeled into the ' $\mathrm{B}$ ' subunit of domain I based on its observed mode of binding in the ' $A$ ' subunit (10). The resulting structure, of domain III with NADP bound interacting with a dimer of domain I with NAD modeled into the site adjacent to NADP, provides a basis for detailed consideration of the roles of the conserved residues in both domains during hydride transfer, and the requirements for conformational changes during proton translocation (11).

In this paper, we report an independent structure determination of domain I ( $\alpha 1$ subunit) of $R$. rubrum $\mathrm{TH}$ in the same crystal form with two dimers in the asymmetric unit.
High-resolution structures have been determined in the absence of $\mathrm{NAD}(\mathrm{H})$ and with $\mathrm{NADH}$ bound, and singlecrystal spectrophotometry was used to confirm the presence of NADH. These new structures, considered together with the domain I structures with NAD bound $(9,10)$, provide a complete picture of the states of domain I with respect to $\mathrm{NAD}(\mathrm{H})$ binding. The structures are compared to the NADbound form (9), and evaluated in terms of the observed interactions with domain III (10) and the proposed mechanism for the hydride transfer reaction (11). The unique conformation and interactions for NADH are established, and comparison of the free and bound states implies conformational changes required for binding nucleotide. Further, comparison of the NADH- and NAD-bound forms reveals conformational changes of conserved residues in the active site, in particular Arg127 and Gln132, which are correlated with the reduced state of the nicotinamide. The results are supported by the presence of multiple copies of the domain I subunit in the asymmetric unit of the crystals in this study and in the structures of the NAD complex $(9,10)$. Together, the results provide insight into the conformational changes associated with the hydride transfer reaction, which is coupled to proton pumping in $\mathrm{TH}$.

\section{EXPERIMENTAL PROCEDURES}

Protein Preparation and Crystallization. The soluble $\alpha 1$ subunit (domain I) of $R$. rubrum transhydrogenase was expressed and purified as previously described (6). The selenomethionine (SeMet) derivative was expressed according to the procedure of Doublié (12) and purified as for domain I. Crystals of domain I are rhombic plates of approximate dimensions $0.3 \times 0.3 \times 0.05 \mathrm{~mm}$ and were grown at $4{ }^{\circ} \mathrm{C}$ by vapor diffusion from a $22 \mathrm{mg} / \mathrm{mL}$ protein solution in $10 \mathrm{mM}$ Tris- $\mathrm{HCl}, \mathrm{pH} 8.0,10 \mathrm{mM}$ ammonium sulfate, $1 \mathrm{mM}$ dithiothreitol, and $0.5 \mathrm{mM}$ PMSF. The reservoir solution contained $18 \% \mathrm{w} / \mathrm{v}$ mPEG $2 \mathrm{~K}$ in $100 \mathrm{mM}$ Tris- $\mathrm{HCl}, \mathrm{pH} 7.5$, and $1 \mathrm{mM}$ magnesium acetate, and was mixed in equal volume $(3 \mu \mathrm{L})$ with the protein solution. The crystals appeared in 3-5 days. They were monoclinic, space group $P 2_{1}$, with two domain I dimers in the asymmetric unit (Table 1). Crystals of NADH-bound domain I were grown under the same conditions (aerobically) with inclusion of 5 mM NADH (Sigma) in the protein solution. These crystals appeared in $\sim 1$ week and were similar in appearance except for being distinctly thicker $(\sim 0.1 \mathrm{~mm})$. Crystals of the NAD complex (9) were grown using these conditions and $5 \mathrm{mM}$ NAD (Sigma).

Single-Crystal Microspectrophotometry. UV-visible spectra of crystals of $\mathrm{TH}$ domain I, with and without $\mathrm{NAD}(\mathrm{H})$ cofactors bound, were measured to probe the presence and oxidation state of NADH in the crystal. Crystals were grown under the same conditions as used for X-ray data collection. Single crystals were transferred to a cryoprotectant solution of $20 \%$ v/v PEG 400 for native (apo) domain I, and $25 \%$ v/v MPEG 550 for domain I bound to NAD $(H)$, in a synthetic mother liquor consisting of $18 \% \mathrm{w} / \mathrm{v}$ mPEG $2 \mathrm{~K}, 100 \mathrm{mM}$ Tris- $\mathrm{HCl}, \mathrm{pH} 7.5$, and $1 \mathrm{mM}$ magnesium acetate. Prior to freezing, the crystals were soaked for $>10 \mathrm{~min}$, to wash out excess $\mathrm{NAD}(\mathrm{H})$ and other reagents not specifically bound to protein molecules in the crystal. The microspectrophotometer is mounted on an optical bench and equipped with a Mercury-Xenon arc lamp light source, optical components 


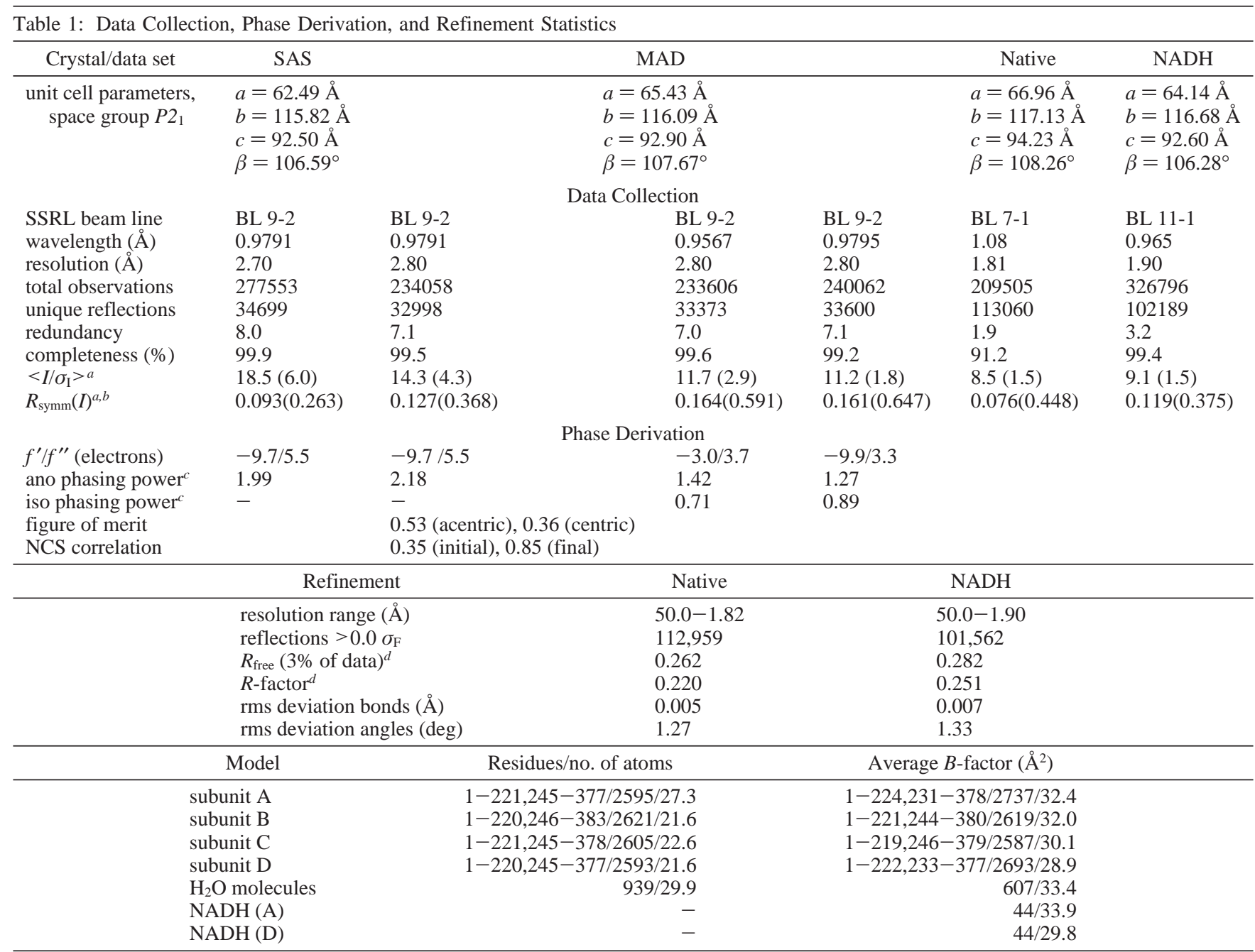

${ }^{a}$ Values in parentheses correspond to the highest resolution shell. ${ }^{b} R_{\text {symm }}=100 \times \Sigma_{h} \Sigma_{j}\left|I_{h j}-I_{h}\right| / \Sigma_{h} \Sigma_{j} \mathrm{I}_{h j}$ where $I_{h}$ is the weighted mean intensity of the symmetry-related reflections $I_{h j} .{ }^{c}$ Isomorphous and anomalous phasing power is the ratio of the calculated heavy atom structure amplitude to the lack of closure. ${ }^{d} R=\Sigma|| F_{\mathrm{obs}}|-| F_{\text {calc }}|| / \Sigma\left|F_{\mathrm{obs}}\right|$, where $\left|F_{\mathrm{obs}}\right|$ and $\left|F_{\text {calc }}\right|$ are the observed and calculated structure factor amplitudes, respectively.

to focus the incident beam to $5 \mu \mathrm{m}$, and a linear CCD array detector. Crystals were mounted on nylon loops, transferred to a goniometer head, positioned with translation stages, viewed with a binocular microscope, and maintained at 100 $\mathrm{K}$ with a liquid $\mathrm{N}_{2}$ supplied cryo-stream. Spectra were measured with the incident beam approximately normal to the face of the platelike crystals. Spectra were collected in the wavelength range $260-700 \mathrm{~nm}$. As each crystal specimen had a different thickness, it was necessary to adjust the incident beam intensity and count time to obtain similar baseline absorbance in the visible region $(400-700 \mathrm{~nm})$ of the spectrum, and also to observe the absorbance at $\sim 340$ $\mathrm{nm}$ for crystals containing NADH. Consequently, a separate reference spectrum was measured at the same incident intensity and count time for each sample.

Data Collection. For X-ray data collection, crystals were flash-frozen at $100 \mathrm{~K}$ using a cryoprotectant of $20 \% \mathrm{v} / \mathrm{v}$ PEG400 (native and SeMet domain I) or 25\% v/v MPEG550 (NADH-bound domain I) in synthetic mother liquor as described above. Data were collected at Stanford Synchrotron Radiation Laboratory (SSRL) beam lines and processed with CCP4 programs (13) (Table 1). For each data set, it was necessary to screen 10-20 crystals due to variable mosaicity in the diffraction observed when the X-ray beam was normal to the thin edge of the plates.
Structure Solution. Experimental phases were derived by a combination of SAS and MAD methods using the SeMet derivative. A redundant SAS data set (Table 1) collected at the Se absorption edge was used to locate 56 of 60 Se sites in the asymmetric unit with the program $\mathrm{SnB}$ (14). For this calculation, low-resolution data to $52.0 \AA$ A spacings were included, the data were scaled, normalized, and filtered with Drear (15), and recommended options in $\mathrm{SnB}$ were employed. The distribution of trial solutions was bimodal (Rbest 0.473 vs Rworst 0.585 ) with $1.2 \%$ of solutions being correct. The Se sites for the best solution were refined using the program Sharp (16), and protein phases were calculated with Sharp using the SAS and three-wavelength MAD data sets (Table 1). The resulting electron density map was interpreted in terms of $\alpha$-helical segments within the four independent subunits in the asymmetric unit in order to define noncrystallographic symmetry (NCS) operators. The electron density was then 4-fold-averaged and solvent-flattened using the program DM (17) (Table 1). The averaged electron density was used for chain tracing and model building using Xfit (18).

Refinement. Four copies of the domain I model were positioned in the asymmetric unit by molecular replacement and refined by simulated annealing against the native data (Table 1) using the program CNS (19). The model for the 


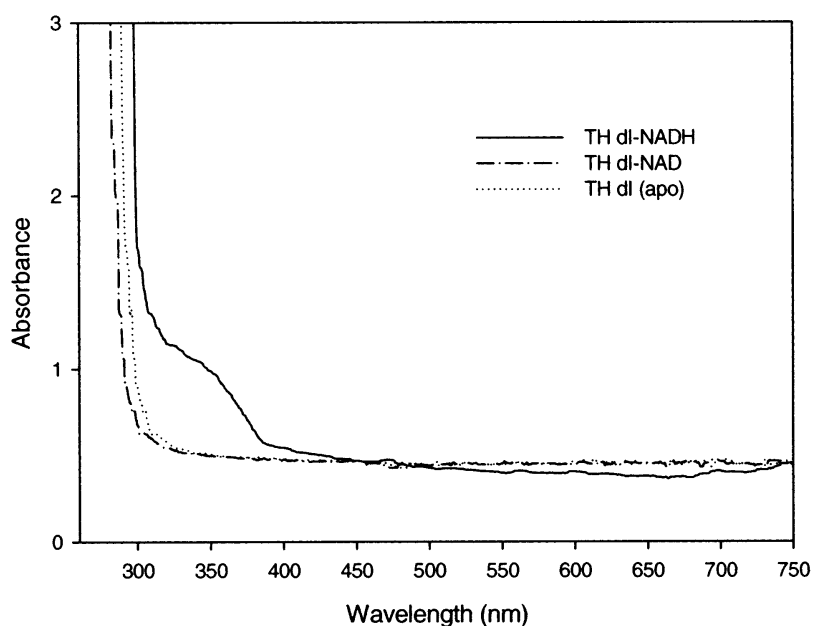

FIGURE 1: UV-visible spectra of single crystals of domain I of $R$. rubrum transhydrogenase grown with NADH (solid line), with NAD (dashed line), and in the absence of added cofactor (dotted line). Spectra were recorded as described under Experimental Procedures for crystals at $100 \mathrm{~K}$. Prior to freezing, crystals were back-soaked in cryoprotectant to remove excess $\mathrm{NAD}(\mathrm{H})$. The dihydronicotinamide moiety of NADH exhibits an absorbance peak at $\sim 340 \mathrm{~nm}$.

native protein was subsequently refined against the data for NADH-bound domain I using rigid-body and simulated annealing refinement with CNS (Table 1). NADH molecules were interpreted in unbiased difference electron density and included in the model. Both the native and NADH-containing domain I models were subjected to multiple iterations of adjustment and editing in $\sigma_{\mathrm{A}}$-weighted $2\left|F_{\mathrm{o}}\right|-\left|F_{\mathrm{c}}\right|$ maps with inclusion of $\mathrm{H}_{2} \mathrm{O}$ molecules and further refinement. Statistics for the final models are included in Table 1. Including all four subunits of the native domain I structure, $99.8 \%$ of residues are within most favored or allowed regions of the Ramachandran plot; for the NADH-bound domain I structure, this is $99.0 \%$ of residues. Coordinates for the native and NADH-bound domain I structures have been deposited in the PDB with accession codes 1L7D and 1L7E, respectively.

\section{RESULTS}

NADH-Containing Crystals. To confirm that $R$. rubrum TH domain I crystals grown aerobically from $5 \mathrm{mM} \mathrm{NADH}$ do contain the reduced cofactor, single-crystal UV-visible spectra were measured at $100 \mathrm{~K}$ using a single-crystal microspectrophotometer, as described under Experimental Procedures. Similar spectra were also measured for crystals of the apo- and NAD-containing crystals. As shown in Figure 1 , crystals of the apo- and NAD-containing protein show no absorbance at longer wavelengths beyond the intense protein peak at $280 \mathrm{~nm}$, while a NADH-containing crystal exhibits a distinct peak at $\sim 340 \mathrm{~nm}$. The characteristic absorption of dihydronicotinamide in NADH at $100 \mathrm{~K}$ is at $347 \mathrm{~nm}(20)$. Due to the optical density of the crystals, it was necessary to use higher incident beam intensity and longer counting times, to observe the NADH absorption. Under these conditions, the protein absorption at $280 \mathrm{~nm}$ exceeds the upper limit of detection, so that it has not been possible to determine absorbance ratios at $280 \mathrm{~nm}$ vs $340 \mathrm{~nm}$ for the different crystal specimens and to estimate the relative occupancy of NADH. Nevertheless, a peak at $\sim 340 \mathrm{~nm}$ was consistently observed in all five NADH-containing crystals that were measured, while spectra of six apo- and six NAD- containing crystals, measured at identical incident beam intensity and for similar count times, do not show absorbance at $\sim 340 \mathrm{~nm}$. This confirms the expectation that the cofactor is not oxidized under the aerobic crystallization conditions used. It can be noted that single-crystal absorbance and fluorescence spectra of malate dehydrogenase show that oxidation of NADH in crystals is very slow; i.e., reduced cofactor is observed after months of aerobic storage (20). For domain I, all crystals were frozen for spectrophotometry or data collection within 1-2 weeks of being grown. Finally, because the apo-, NAD-, and NADH-containing crystals are back-soaked to remove excess, unbound reagents, and frozen under very similar cryoprotectant conditions, the singlecrystal spectra confirm the presence of NADH in domain I crystals used for X-ray data collection.

Domain I Structure. We initiated structural studies of transhydrogenase with determination of the bovine domain III structure at $1.2 \AA$ resolution (7). Subsequently, we obtained crystals of $R$. rubrum domain I ( $\alpha 1$ subunit), collected native data, and MAD and SAS data sets (Table 1). In the meantime, Buckley et al. reported the structure of R. rubrum domain I with NAD bound at $2.0 \AA$ resolution in the same monoclinic crystal form (9). At this point, we had determined 56 of the 60 Se positions in the SeMet derivative (Table 1). Because the coordinates for the NAD complex were on hold for 1 year (PDB entry 1F8G), we continued with the interpretation of the structure independently. However, knowledge of the overall fold of domain I, in particular that the structure contained long $\alpha$-helices (Figure 1 of ref 9), facilitated interpretation of the initial electron density maps and definition of the NCS operators. The 4-fold averaged map resulted in excellent electron density, and was used for model building. We carried out refinement of the domain I native, i.e., 'apo', structure at $1.8 \AA$ resolution (Table 1). Subsequently, we determined the structure of the NADH complex at 1.9 A resolution (Table 1; Figure 2). The domain I models derived from the independent structure determinations are overall very similar with rms differences in $\mathrm{C} \alpha$ positions of $0.35-0.55 \AA$ (below). Together, the structures with NAD bound $(9,10)$, with NADH bound, and in the absence of dinucleotide substrate provide the basis for observing conformational states relevant to the function of domain I in the holoenzyme.

For purposes of discussion, the $\alpha 1$ subunit of $R$. rubrum TH is considered domain I of transhydrogenase. As observed for the NAD complex (9), the crystal structure contains two domain I dimers in the asymmetric unit; in each, a local 2 -fold axis relates the individual subunits, which are denoted $\mathrm{A}$ and $\mathrm{B}$ in one dimer, and $\mathrm{C}$ and $\mathrm{D}$ in the other (Figure 3). The four crystallographically independent subunits are designated $\mathrm{dI}(\mathrm{A}), \mathrm{dI}(\mathrm{B}), \mathrm{dI}(\mathrm{C})$, and $\mathrm{dI}(\mathrm{D})$ (Figure 3 inset). The $40 \mathrm{kDa}$ domain I protein is comprised of two distinct, separately folded components or subdomains ("domains" in terms of the protein architecture); these are designated dI.1 and dI.2 (Figure 3 inset) $(9,10)$. The protein architecture of the subunit and the arrangement of the dimer are as observed for the NAD complex (9) and in cocrystals with domain III (10). Briefly, the dI.1 and dI.2 components each contain a central $\beta$-sheet and flanking $\alpha$-helices with the topology of nucleotide binding folds. The dI.1 component is comprised of residues 1-137 and 328-384, and the dI. 2 component of residues 138-327 (9). The $\beta$-sheet of dI.1 contains strands 

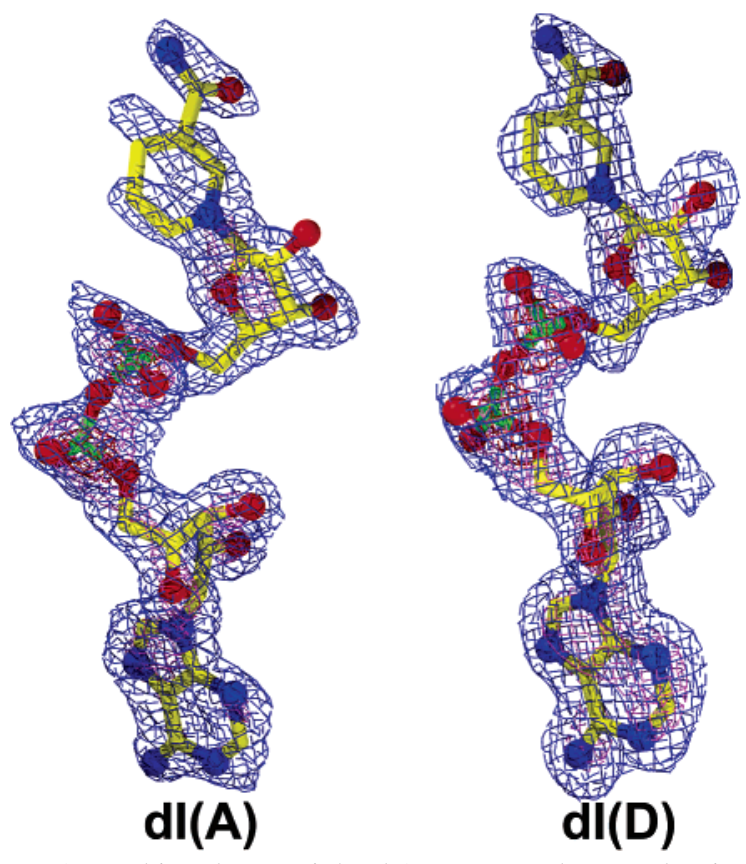

Figure 2: Unbiased $\sigma_{\mathrm{A}}$-weighted $2\left|F_{\mathrm{o}}\right|-\left|F_{\mathrm{c}}\right|$ electron density for NADH in the dI(A) and dI(D) subunits in the crystal structure of domain I ( $\alpha 1$ subunit) of $R$. rubrum transhydrogenase at $1.9 \AA$ resolution. The map is contoured at 1,3 , and $5 \sigma$. Atoms are colored $\mathrm{C}$ (yellow), $\mathrm{N}$ (blue), $\mathrm{O}$ (red), and $\mathrm{P}$ (green). This figure was prepared with Xfit (18) and Molw/Showcase (21).

arranged in the order $\mathrm{c}_{1} \mathrm{~b}_{1} \mathrm{a}_{1} \mathrm{~d}_{1} \mathrm{e}_{1} \mathrm{f}_{1} g_{1} \mathrm{~h}_{1}$ where only strand $g_{1}$ is antiparallel to the others; the $\beta$-sheet of dI. 2 contains strands arranged in the order $\mathrm{c}_{2} \mathrm{~b}_{2} \mathrm{a}_{2} \mathrm{~d}_{2} \mathrm{e}_{2} \mathrm{~g}_{2} f_{2}$ where only strand $f_{2}$ is antiparallel to the others. The $\beta$-sheets are linked by a pair of long $\alpha$-helices that connect strands $\mathrm{f}_{1} \rightarrow \mathrm{a}_{2}$ (helix residues 135-155) and $\mathrm{g}_{2} \rightarrow g_{1}$ (helix residues 327-346); these helices lie to one side of the subunit, and the C-terminal ends of the $\beta$-strands are oriented toward a cleft separating dI.1 and dI.2. The long $\mathrm{f}_{1} \rightarrow \mathrm{a}_{2}$ helix is extended at its C-terminus by an antiparallel $\beta$-loop (residues $160-173$ ) that forms extensive contacts with the 2-fold related subunit (Figure 3). Overall, the architecture of the dimer is such that interactions between dI.1 and dI.2 in 2-fold related subunits are more extensive than between $\mathrm{dI} .1$ and $\mathrm{dI} .2$ in the same subunit.

NADH Binding Site. NADH is bound to one subunit (A, D) of each dimer (Figures 2 and 3). The interactions between domain I and NADH (Figure 4) are similar to those observed for NAD $(9,10)$ except in the region of the nicotinamide, where distinct conformational differences occur and where the nicotinamide is exposed in the cleft between dI.1 and dI.2 (below). The adenosine monophosphate (AMP) moiety of the dinucleotide binds across the C-terminal ends of the $\beta$-strands of dI.2 in typical fashion for nucleotide binding proteins. A $\beta \alpha \beta$ element within this parallel $\beta$-sheet makes contacts to ribose and phosphate via a Gly179-Val-Gly181 motif (strand $\mathrm{a}_{2}$ ) and Asp202 (strand $\mathrm{b}_{2}$ ), which hydrogen bonds to both $2^{\prime}$ - and 3'-hydroxyl groups, providing specificity for NAD vs NADP. The N-terminal dipole of the $\alpha$-helix in this $\beta \alpha \beta$ element (residues 181-193) is directed toward both phosphates. An adjacent conserved residue, Arg204, hydrogen bonds with each ribose of NADH (Figure 4). Adenine of the AMP moiety is buried within the hydrophobic core of dI. 2 with complementary interactions of N1 and N6 with $\operatorname{Gln} 247$.
The nicotinamide nucleoside portion of NADH is exposed in the cleft between the domains where the nicotinamide occupies a pocket at the base of the long $\mathrm{f}_{1} \rightarrow \mathrm{a}_{2} \alpha$-helix that extends toward the 2-fold axis and links dI.1 to dI.2 (Figure 3 ). The open pocket is formed by a 1.5-turn 310 -helix following the $f_{1}$ strand, and residues at the $\mathrm{N}$-terminal end of the $\alpha$-helix (residues 126-138) (Figure 4). As noted for the NAD complex (9), six of these residues are conserved in TH domain I sequences, i.e., Pro126, Arg127, Ala131, Gln132, Asp135, and Ser138. Arg127 is extended across the binding pocket and interacts with the nicotinamide nucleotide phosphate; Gln132 interacts with the nicotinamide side chain; and Asp135 and Ser138 are within 3.2-3.7 $\AA$ of nicotinamide atoms. Arg127 and also Arg204 are in contact with a loop of residues 224-240 having a $\beta$-turn at Gly233-Gly234. Three residues in this loop closest to the arginines, Gly234, Tyr235, and Ala236, are also conserved. This loop, together with the open pocket of conserved residues and the $\beta \alpha \beta$ element, defines the nicotinamide binding site. However, this loop is flexible, being disordered in six copies of the subunit in the absence of NADH, but significantly more ordered when NADH is bound (Figure 4; Table 1). This loop is also observed to be mobile in crystals of domain I with NAD bound, but it becomes defined when Tyr235 hydrogen-bonds to NAD or $\operatorname{Arg} 127(9,10)$.

Three reagents, FSBA, DCCD, and EEDQ, have been used to identify functionally important amino acids in domain I of transhydrogenase. In particular, these reagents chemically modify residues in the bovine enzyme corresponding to $R$. rubrum residues Tyr235, Gln247, and Asp222 (1). Because the enzyme is protected from these inhibitory modifications by $\mathrm{NAD}(\mathrm{H})$, these residues are implicated in the $\mathrm{NAD}(\mathrm{H})$ binding site. Consistent with this, Gln247 is in direct contact with adenine of NADH and Tyr235 is part of the binding site (Figure 4); modification of Asp222 might be explained by an effect on the adenine binding pocket in dI.2.

The NADH binding site, which involves minimal contacts to the nicotinamide, leaves it exposed to solvent in the cleft between dI.1 and dI.2; however, the conformation of NADH is well-defined and very similar in both subunits $\mathrm{dI}(\mathrm{A})$ and dI(D) (Figure 5). In accord with the known stereospecificity of the reaction $(1-4)$ the pro- $R$ face (A-face) of the nicotinamide at $\mathrm{C} 4$ is fully exposed while Arg127 occludes the pro- $S$ face (B-face), which is 3.9-4.9 $\AA$ from the nearest side chain atom of Arg127. The anti conformation about the glycosidic bond (Figures 4 and 5) is consistent with the observed tendency for enzymes exhibiting pro- $R$ stereochemistry to adopt this conformation (23). The anti stereochemistry for NADH is supported by hydrogen bonds involving $\mathrm{N} 7$ and $\mathrm{O} 7$ of the nicotinamide carboxamide group with the carbonyl of Ile128 and the side chain of Gln132, respectively. These interactions appear to ensure the correct orientation of the reduced nicotinamide in its exposed binding site.

Comparison of Subunits. The crystal structures of the native and NADH-bound $\alpha 1$ subunit (Table 1) provide eight crystallographically independent copies of domain I. Comparison of these provides insight into the mobility of side chains associated with the binding and release of the dinucleotide substrate (product) (eq 1). Least-squares superposition of $350 \mathrm{C} \alpha$ atoms in common among the 8 copies results in rms deviations of $0.36-0.60 \AA$. The NADH 


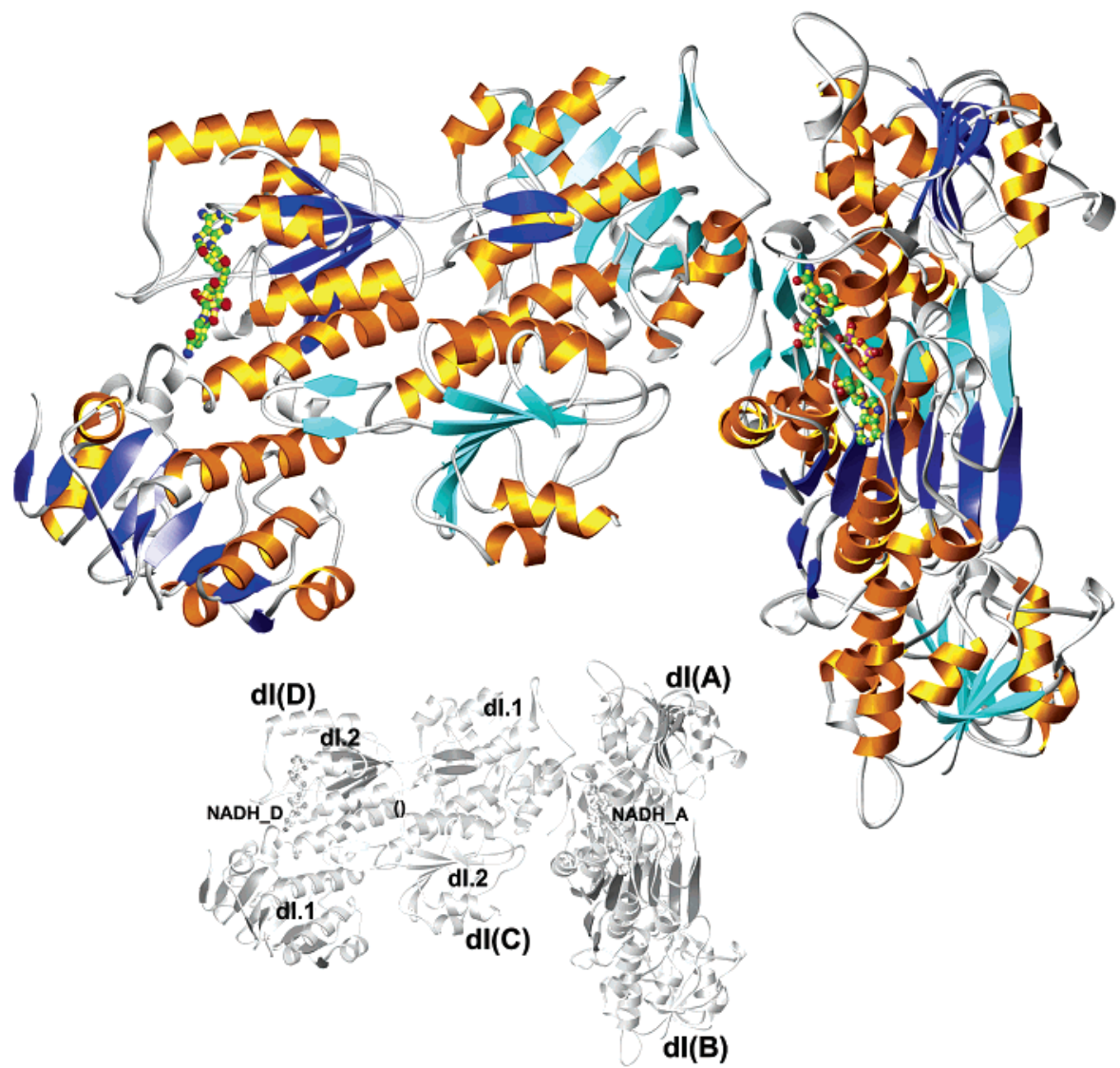

FIGURE 3: Crystal structure of the NADH-bound form of domain I ( $\alpha 1$ subunit) of $R$. rubrum transhydrogenase. The contents of the asymmetric unit of the monoclinic crystals are shown viewing along the local 2-fold axis of the CD dimer (left). The 2-fold axis is indicated by parentheses in the inset figure. The local 2-fold axis of the AB dimer (right) is nearly orthogonal and is in the plane of the figure. The subunits are denoted $\mathrm{dI}(\mathrm{A}), \mathrm{dI}(\mathrm{B}), \mathrm{dI}(\mathrm{C})$, and $\mathrm{dI}(\mathrm{D})$; each is comprised of two folded components denoted dI.1 and dI.2 (see inset). The protein structure is represented with gold coils for $\alpha$-helices, and blue [dI(A), dI(D)] or cyan $[\mathrm{dI}(\mathrm{B}), \mathrm{dI}(\mathrm{C})]$ ribbons for $\beta$-sheets. $\mathrm{dI}(\mathrm{A})$ and dI(D) each bind one NADH molecule (space-filling models). This figure was prepared with Ribbons (22).

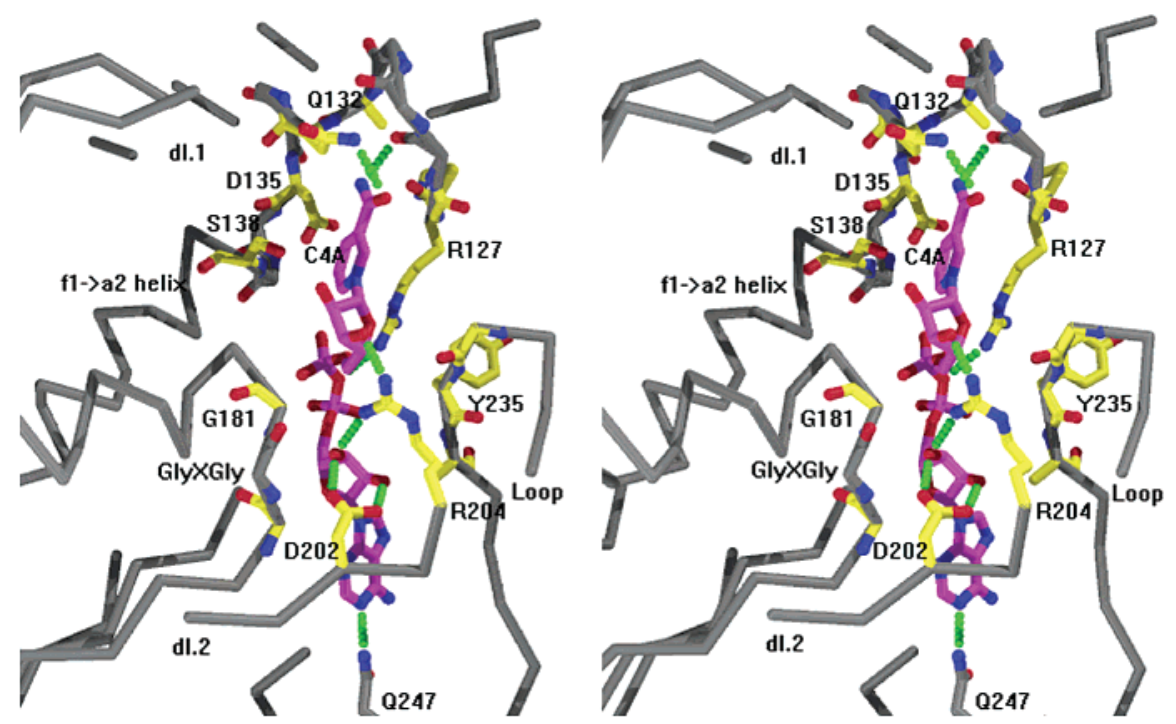

FIGURE 4: Stereo figure of the NADH binding site in the dI(A) subunit. The site is comprised of residues at the N-terminal end of a $\alpha$-helix connecting dI. 1 and dI.2 ( $\mathrm{f}_{1} \rightarrow \mathrm{a}_{2}$ helix), including a short $3_{10}$-helix, a $\beta \alpha \beta$ nucleotide binding motif in dI.2 (including GlyXGly), and two loops identified by Arg204 and Tyr235. Residues conserved in TH domain I sequences are yellow, C $\alpha$ atom positions are linked in gray, $\mathrm{NADH}$ is magenta, and $\mathrm{N}$ and $\mathrm{O}$ atoms are blue and red, respectively. Hydrogen bonds to NADH are indicated by green dotted lines. Amino acids are indicated by the single-letter code (D, Asp; G, Gly; Q, Gln; R, Arg; S, Ser; Y, Tyr). This figure and Figures 5 and 6 were prepared with Molw/Showcase (21).

conformation in the $\mathrm{A}$ and $\mathrm{D}$ subunits occupied by the dinucleotide is very similar $(<0.25 \AA$ difference for 44 atoms), and similar hydrogen bonds involving $\mathrm{N} 7$ and $\mathrm{O} 7$ are formed in each case (Figure 5). This suggests that the observed conformation is relevant to hydride transfer when domain I interacts with domain III in the intact enzyme.

In contrast, there is significant variability in the six copies of domain I in the absence of NADH. The three residues 

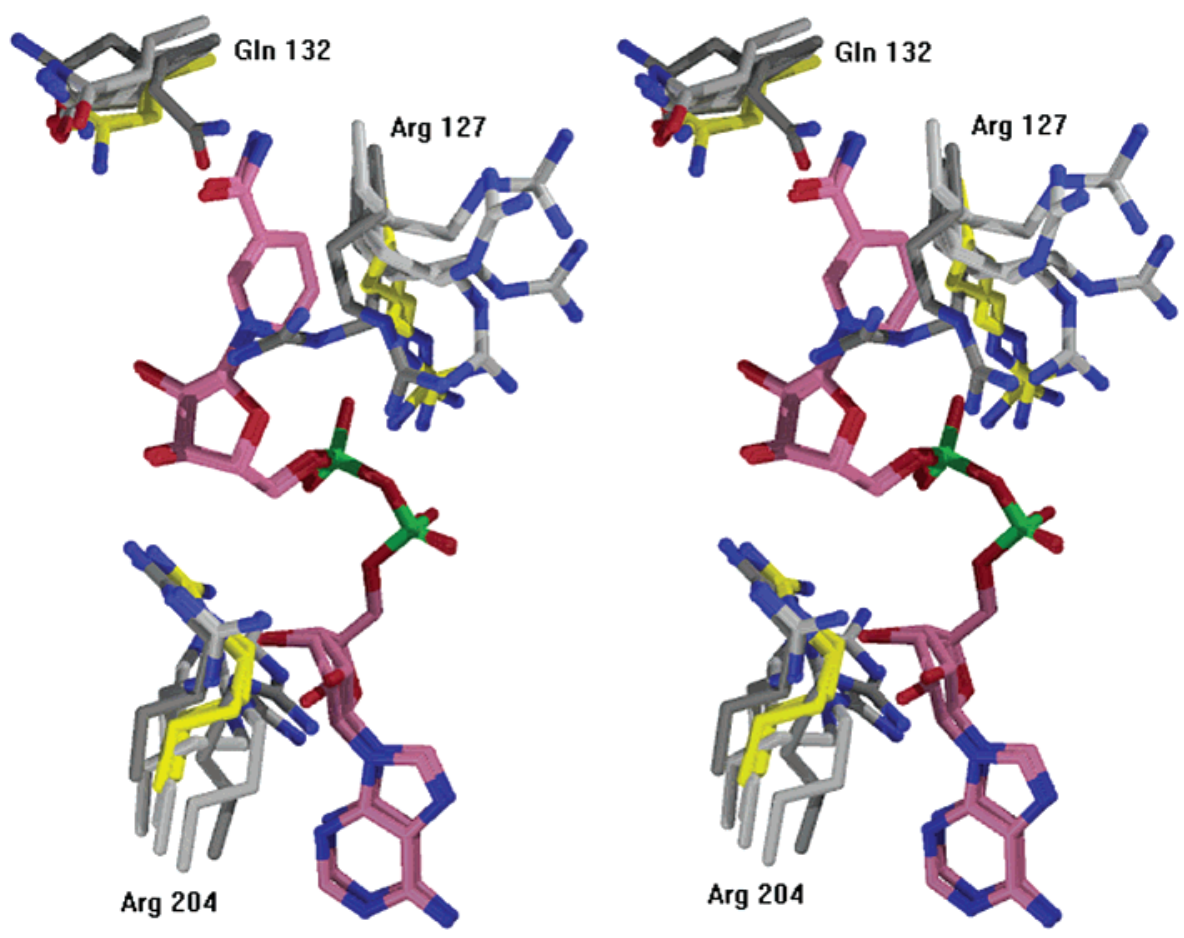

FIGURE 5: Stereo figure showing the NADH binding site in eight superposed copies of domain I with and without NADH bound. The bound conformation of $\mathrm{NADH}$ in the $\mathrm{dI}(\mathrm{A})$ and $\mathrm{dI}(\mathrm{D})$ subunits is virtually identical. The three conserved residues that display the greatest variation in position are shown; their conformations when interacting with NADH are indicated in yellow; the remaining six copies are indicated in gray. The $B$-factors of these side chains vary: Arg127, 27-44 $\AA^{2}$; Gln132, 18-30 $\AA^{2} ;$ Arg $204,25-55 \AA^{2}$.

with the greatest variation are $\operatorname{Arg} 127, \mathrm{Gln} 132$, and $\operatorname{Arg} 204$ (Figure 5). In particular, the location of the Arg127 side chain varies up to $9.6 \AA$, and that of $\operatorname{Arg} 204$ up to $6.8 \AA$. The superposition of occupied and vacant binding sites provides a snapshot of how these residues may change conformation during binding. When NADH is present, these side chains form optimal interactions, implying that they play an important role in positioning NADH. Presumably, open conformations of Arg127 and Arg204, as well as of the flexible loop of residues 224-240 (Figure 4), would be required for $\mathrm{NAD}(\mathrm{H})$ exchange. The role of the flexible loop carrying Tyr235 in binding or release of $\mathrm{NAD}(\mathrm{H})$ has been suggested based on the structure of the NAD complex (9) and is supported by NMR and mutagenesis data (3). Participation of this loop in $\mathrm{NAD}(\mathrm{H})$ binding is also consistent with inhibition by FSBA which modifies Tyr245 of bovine TH (Tyr235, Figure 4) (5). Together the implied conformational changes could be expected to facilitate binding and release of $\mathrm{NAD}(\mathrm{H})$ during hydride exchange in TH.

Comparison of Dimers. Intact TH is dimeric and manifests half-of-the-sites reactivity; i.e., the enzyme binds $1 \mathrm{~mol}$ of $\left[{ }^{14} \mathrm{C}\right] \mathrm{NADH}$ or $\left[{ }^{14} \mathrm{C}\right] \mathrm{NADPH}$ per dimer (5). This result is supported by the structure where domain I crystallized in the presence of NADH contains one NADH per dimer in each of two dimers in the crystallographic asymmetric unit (Figures 2 and 3). Together, the NADH-bound and 'apo' structures (Table 1) provide four crystallographically independent copies of the domain I dimer. We compared the $\mathrm{AB}$ and $\mathrm{CD}$ dimers with and without NADH bound to address a possible allosteric mechanism accounting for the half-of-thesites reactivity. Least-squares superposition of the four dimers results in rms deviations for $670 \mathrm{C} \alpha$ atoms of $0.52,0.92$, and $0.98 \AA$. Comparison of the $\mathrm{AB}$ vs $\mathrm{CD}$ dimers about their local 2-fold axes, such that the NADH-bound A and D subunits are superposed, indicates a significant $(\sim 1 \AA)$ contraction around NADH. This contraction has also been observed for the NAD complex of domain I (9). Flexible portions of dI.1 and dI.2 pack closer to the nucleotide, including residues $126-136$, the loop following the $b_{2}$ strand (Arg204), the loop following the $\mathrm{d}_{2} \beta$-strand (residues 267271), and the highly mobile loop (residues 232-236) (Figure 4). In addition, conserved residues 166-168 at the tip of the extended $\beta$-hairpin in the 2 -fold-related subunit move toward NADH.

However, despite the observed contraction around the NAD and NADH upon binding, the structures do not show systematic positional shifts within the 2-fold related subunit or $\mathrm{NAD}(\mathrm{H})$ site when the independent copies of the $\mathrm{AB}$ and $\mathrm{CD}$ dimers are superposed. Consequently, the half-of-thesites binding exhibited by domain I does not appear to be due to a net conformational difference in unoccupied $\operatorname{NAD}(\mathrm{H})$ sites, i.e., allostery. Perhaps flexibility necessary for binding in a 2-fold related site is restricted when one $\mathrm{NAD}(\mathrm{H})$ is bound. This would not be surprising in view of the intimate association of subunits, i.e., the interdigitating extended $\beta$-hairpins, the packing of helices from each subunit about the local 2-fold, and the contacts of the $\beta$-hairpin with these helices (Figure 3). Interactions across the dimer interface, influencing conformation in the 2-fold subunit, have been envisaged as part of the 'alternating site, binding change mechanism' for TH (11). In the context of this mechanism, an explanation for the half-of-the-sites reactivity of TH is that the entire polypeptide, i.e., dI-dII-dIII, functions as a single cooperative unit, so that only half of the dimeric enzyme can bind $\mathrm{NAD}(\mathrm{H})$ at a given time (11).

$N A D H$ vs NAD Binding. In Figure 6 five independent copies of $\mathrm{NAD}(\mathrm{H})$-bound structures are superposed: these 

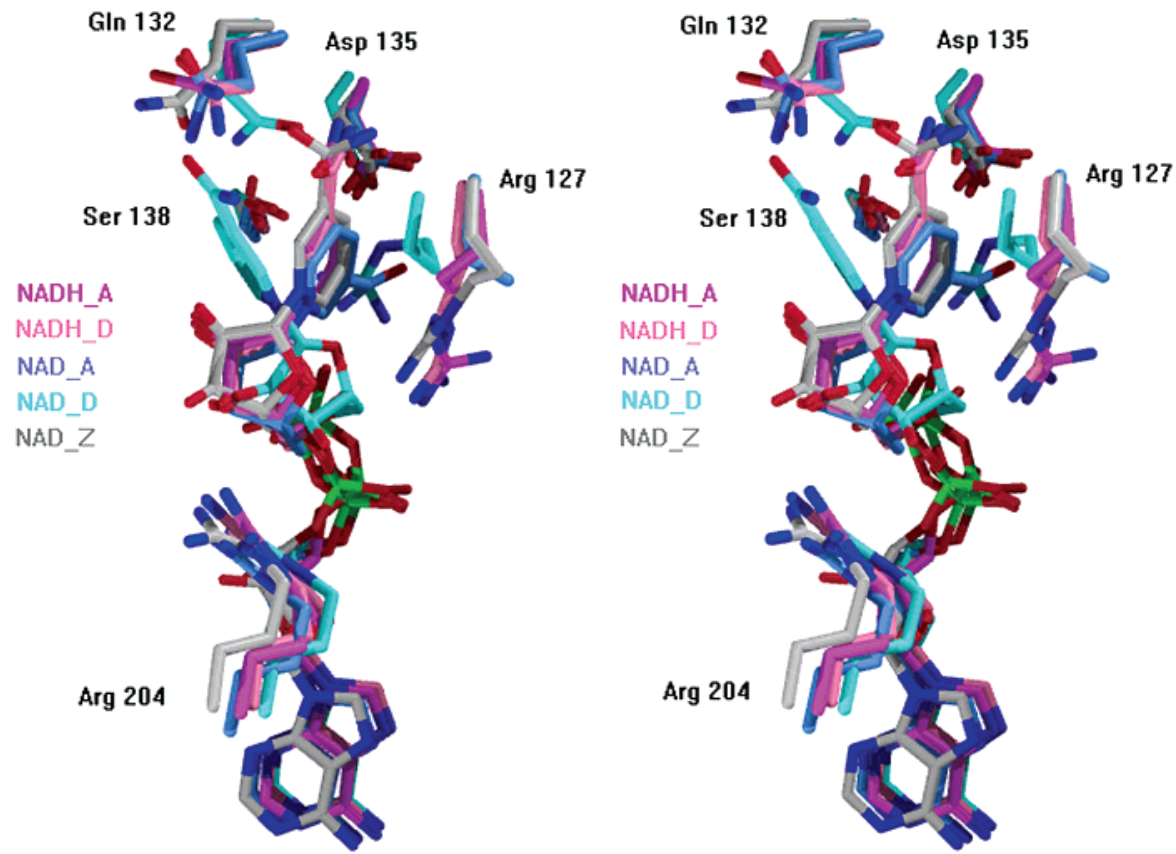

FIGURE 6: Stereo figure showing the NAD(H) binding site in five superposed copies of domain I with NADH bound ('NADH_A', 'NADH_D') or NAD bound ('NAD_A', 'NAD_D', ref 9, PDB code 1F8G; and 'NAD_Z', ref 10, PDB code 1HZZ). The side chains of five conserved residues and the $\operatorname{NAD}(\mathrm{H})$ dinucleotides are shown. Carbon atoms in the five structures are colored according to the legend at the left; $\mathrm{N}$, blue; $\mathrm{O}$, red; $\mathrm{P}$, green.

are for NADH in the A and D subunits ('NADH_A', 'NADH_D') (Table 1); NAD in the corresponding A and D subunits in the same crystal form ('NAD_A', 'NAD_D') (9); and NAD in the A subunit of the heterotrimeric complex (denoted as 'NAD_Z') (10). Weak electron density for NAD was observed in the $\mathrm{B}$ and $\mathrm{C}$ subunits of the domain I dimer (9), and was modeled as ADPribose without nicotinamide (PDB code 1F8G). Weak electron density was also observed in the B subunit of the heterotrimeric domain I:domain III complex (10); this electron density was not directly interpreted (PDB code 1HZZ), but NAD was modeled into this site in order to study the interaction with domain III (11). For the purposes of this comparison, we focus on models of $\mathrm{NAD}(\mathrm{H})$ fit to observed electron density.

The oxidized and reduced nucleotides are compared by superposition of the domain I protein structures. With reference to the NADH_A-bound subunit, the rms differences for all $\mathrm{C} \alpha$ atoms in the NADH_D, NAD_A, NAD_D, and NAD_Z subunits are $0.44,0.35,0.55$, and $0.66 \AA$, respectively. The conformation of NADH in the 'NADH_A' and 'NADH_D' subunits is very similar (Figure 5). However, the NADH conformation differs very significantly compared to NAD (Figure 6). For the 'NAD_A' subunit, the nicotinamide is syn and N7 hydrogen-bonds with phosphate; for the 'NAD_D' subunit, the nicotinamide and ribose are shifted and the glycosyl torsion angle, while anti, is significantly rotated; for the 'NAD_Z' subunit, the nicotinamide ring superposes more closely with NADH but the carboxamide side chain is rotated differently. The variable conformations of NAD vs NADH are associated with different interactions with the flexible portions of domain I (9), and further illustrate the flexibility involved in binding and release of $\mathrm{NAD}(\mathrm{H})$ (Figure 5).

One explanation for the variable conformations of NAD vs the similar conformations of NADH is that electrostatic repulsion between NAD and Arg127 causes these groups to be reoriented. For the 'NAD_A' conformation, the nicotinamide interacts with phosphate, and Arg127 is disordered beyond $\mathrm{C} \delta$ (Figure 6) but oriented away as in unbound subunits (Figure 5). For 'NAD_D', the nicotinamide is significantly shifted, and Arg127 interacts with Asp135. For 'NAD_Z', the position of Arg127 is similar to that in the $\mathrm{NADH}$-bound conformation, but the nicotinamide side chain and Gln132 differ somewhat. Thus, both observed NADHbound sites are very similar, but three NAD-bound sites each differ from each other. Hence, electrostatic repulsion appears to play a role in defining the conformation of $\mathrm{NAD}(\mathrm{H})$ and active site residues, although the 'NAD_Z' conformation shows that this is not the only factor. Nevertheless, this comparison of NAD- and NADH-bound states reveals a means by which nicotinamide oxidation state can influence enzyme conformation.

Kinetic experiments have defined two states for bound $\mathrm{NADH}$, one with a $K_{\mathrm{d}}$ of $\sim 30 \mu \mathrm{M}$, and one with a $K_{\mathrm{d}}$ of $\sim 300 \mu \mathrm{M}(24,25)$. These binding constants are interpreted as corresponding to favorable binding when domain I is associated with domain III (with NADP bound) in the 'open' state, and weaker binding when domain III is converted to the 'occluded' state prior to rapid hydride transfer (11). The variable conformations of NAD and NADH are consistent with the two types of $K_{\mathrm{d}}$ values for NADH (11, Figure 6). However, in the absence of an actual complex with domain III, it is not possible to identify the bound state of NADH, as observed in isolated domain I, as corresponding to the high- or low- $K_{\mathrm{d}}$ form.

\section{DISCUSSION}

Transhydrogenase couples proton translocation across a membrane in both mitochondria and prokaryotes with hydride transfer between $\mathrm{NAD}(\mathrm{H})$ and $\mathrm{NADP}(\mathrm{H})$ (eq 1). The hydride transfer is direct and involves the $4 \mathrm{~A}$ position of 


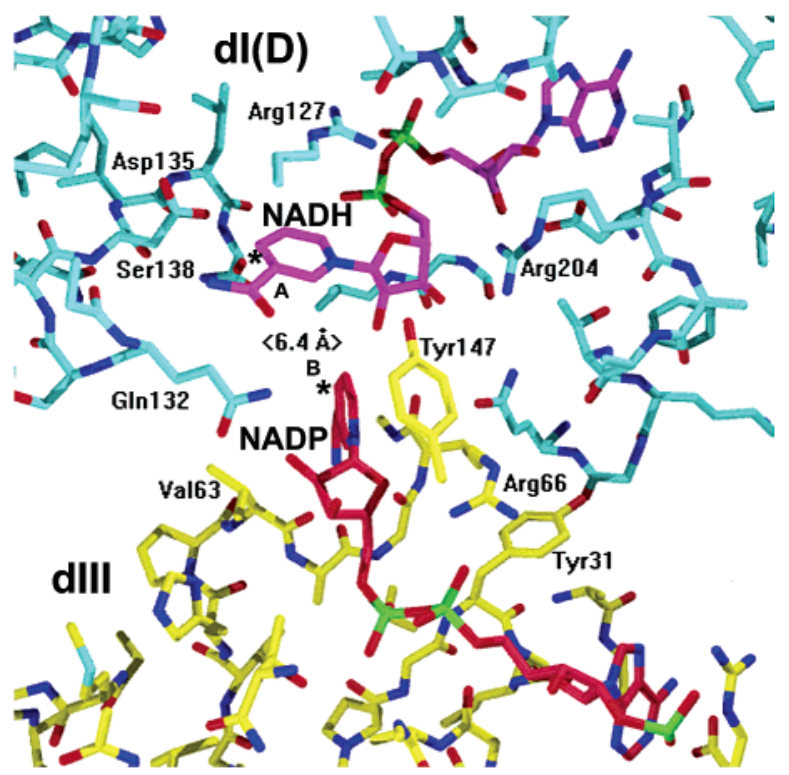

FIGURE 7: Model for the interaction of TH domains involved in hydride transfer. The model shows the position of NADH in domain I with respect to NADP in domain III, and represents the oxidation states in the forward reaction (eq 1). It is based upon superposition of the $\mathrm{dI}(\mathrm{D})$ subunit of the $R$. rubrum domain I:NADH complex (Figure 3) and the bovine dIII:NADP complex (7) onto the corresponding subunits in the heterotrimeric complex of $R$. rubrum domains I and III containing NAD and NADP (PDB code 1HZZ, refs 10,11 ). The heterologous complex of these soluble $R$. rubrum and bovine TH domains is active for hydride transfer (26). The pro- $R$ (A) face of NADH at $\mathrm{C} 4(*)$ and pro-S (B) face of NADP at $\mathrm{C} 4(*)$ are $6.4 \AA$ apart in this model. Several conserved residues in each domain are labeled.

$\mathrm{NAD}(\mathrm{H})$ and the $4 \mathrm{~B}$ position of $\mathrm{NADP}(\mathrm{H})$. Consequently, domains I and III must come into close proximity in the intact enzyme (1-4). Because the difference in the reduction potentials of NADPH/NADP and NADH/NAD is negligible $(\sim 5 \mathrm{mV})$, and all the reactants and the products occur on the same side of the membrane, the driving force for outward proton translocation, coupled to the reverse reaction, must be the difference in the binding energies of the reactants (NADPH and NAD) and the products (NADP and NADH). In other words, outward proton translocation is driven via conformational change due to binding of NADPH and NAD vs NADP and NADH $(1,2)$. Conversely, the pmf alters TH conformation when protons are translocated inward in the forward reaction, resulting in increased affinity for NADH and NADP and acceleration of hydride ion transfer from NADH to NADP $(1-4)$.

Interaction of Domains I and III. It is of interest to evaluate the forward reaction, i.e., transfer of hydride from NADH to NADP, in view of the available crystal structures (7-11; Table 1) and the requirement that $\mathrm{NAD}(\mathrm{H})$ and $\mathrm{NADP}(\mathrm{H})$ must come into direct contact. Expressed soluble R. rubrum domain I and bovine domain III cross-react to catalyze transhydrogenation (26); thus, the absence of domain II, which is membrane-intercalated and contains the proton channel, does not prevent association of the soluble domains. Previously, we determined the structure of bovine domain III with NADP bound (7). Because bovine domain III and $R$. rubrum domain I cross-react, and because the structures correspond to the correct oxidation states (NADH, NADP) in the forward reaction (eq 1), we modeled this heterologous complex (Figure 7). The model was constructed based on the crystal structure of the heterotrimeric $R$. rubrum domain I:domain III complex $(10,11)$. The $R$. rubrum subunit dI(D) with NADH bound was superposed onto the B subunit of the heterotrimeric complex (rms deviation $0.63 \AA$ for all $\mathrm{C} \alpha$ atoms). For domain III, the sequences from bovine and $R$. rubrum are $24 \%$ identical and $>40 \%$ conserved, and the structures are overall very similar $(7,8)$. The bovine domain III with NADP bound was superimposed onto domain III of the $R$. rubrum heterotrimeric complex (rms deviation 0.89 $\AA$ for $174 \mathrm{C} \alpha$ atoms). As expected, the resulting model shows overall shape complementarity of domains I and III and conserved residues in close proximity (Figure 7), as observed for the all $R$. rubrum heterotrimeric complex $(10,11)$.

In structures of enzymes containing NADH or NADPH, the dihydronicotinamide ring stacks parallel with substrate or flavin, and the $\mathrm{C} 4$ atom is within $3.0-3.5 \AA$ of the hydride acceptor, consistent with direct hydride transfer (27-30). Except for glutathione reductase (30), these enzymes follow the correlation that transfer from the pro- $R$ (A) face of the dihydronicotinamide is favored when the glycosyl torsion angle is anti while transfer from the pro-S (B) face is favored when it is syn (23). In TH hydride transfer occurs between the A-face of $\operatorname{NAD}(\mathrm{H})$ and B-face of $\operatorname{NADP}(\mathrm{H})(1-4)$. In accord with the preferred stereochemistry, the glycosyl torsion angles for NADH and NADP are anti and syn (Figure 7 ), respectively. However, the $\mathrm{C} 4$ atoms of the nicotinamide rings of NADH and NADP are far apart $(6.4 \AA)$ and not coplanar (Figure 7). A similar result was obtained when NAD was modeled into the ' $\mathrm{B}$ ' subunit of domain $\mathrm{I}$ in the heterotrimeric complex, based on consideration of the NAD binding site in the 'A' subunit and rotation of flexible torsion angles to place $\mathrm{NAD}(\mathrm{H})$ adjacent to NADP in domain III $(10,11)$. In this case, the $\mathrm{C} 4$ atoms are $6.5 \AA$ apart and the nicotinamide rings are also significantly nonparallel. Hence, the models for the interaction of NADH and NADP in the domain I:domain III interface are consistent, but as discussed with respect to the heterotrimeric complex (11), it is clear that for direct hydride transfer to occur, which is also very rapid (31), there must be movement of the subunits and/or the nucleotides.

What might be the driving force to make domains I and III interact more closely? It seems possible that electrostatic effects due to nicotinamide net charge, as seen for NADH vs NAD in domain I (Figure 6), could also influence the interaction between domains. In addition to $\operatorname{Arg} 127, \operatorname{Arg} 204$, and Asp135 in domain I, which can exhibit several conformations (Figures 5 and 6), there is a conserved arginine in domain III close to NADP (Arg90 in R. rubrum, ref 11; Arg66, Figure 7). These invariant charged residues are likely to be critical in defining the active conformation $(11,31)$. At the same time, flexible loops in domain I, and loops adjacent to NADP in domain III (loops 'D' with Asp131 and 'E' with Tyr171 in R. rubrum, ref 11; Tyr147 in Figure 7), could alter their conformation to allow closer association of the domains. A docked and active complex could also involve hydrogen bonding of conserved residues, such as Gln132 and Tyr147 (Figure 7), with ribose groups of the cofactor across the domain interface, as seen in the heterotrimeric complex (11).

The driving force for the overall reaction (eq 1) derives from the pmf in the forward direction, and from the difference in binding energy for NADPH and NAD vs NADP 
and NADH in the reverse direction. However, in the absence of domain II, water-soluble recombinant domains I and III interact and catalyze transhydrogenation $(6,25,26)$. Further, the rate of hydride transfer from NADH to NADP in the forward reaction is very fast $(25,31)$. Hence, hydride transfer does not require domain II, and additional movement between domains I and III to form an active complex is not dependent on proton translocation. Nevertheless, alternate conformations of domains I and III must arise as a result of binding oxidized and reduced nicotinamide nucleotides. For example, NADPH has a dramatic effect upon the trypsin sensitivity of TH at two remote sites $(32,33)$, and it alters the rate of chemical modification at the $\operatorname{NAD}(\mathrm{H})$ site in domain I (2,34). NMR data indicate conformational changes in domain III upon NADP reduction (35). Motion of domain III within TH is described in terms of 'open' and 'occluded' states in the 'alternating site, binding change mechanism' (11). Based on kinetic data for the heterotrimeric complex in solution $(24,31)$, the cocrystal structure with NADP bound to domain III, e.g., Figure 7, corresponds to the 'occluded' state (11). Recently, a conserved acidic residue ( $\beta$ Asp213) in an extramembranous loop of domain II of E. coli TH has been found to be required for proton translocation, suggesting a mechanism by which NADPH-induced movement of domain III could affect a $\mathrm{p} K_{\mathrm{a}}$ shift in domain II, which in turn would favor proton translocation (36).

\section{ACKNOWLEDGMENT}

We thank Elizabeth Getzoff for expert advice and for the use of the single crystal microspectrophotometer in her laboratory. We thank Frank von Delft for very generous assistance in the application of computer programs, and Victoria Roberts for considerations in regard to docking. We also thank the staff at the Stanford Synchrotron Radiation Laboratory (SSRL) for their excellent support in the use of beam lines. This work is based on research conducted at SSRL, which is funded by the Department of Energy, Office of Basic Energy Sciences. SSRL beam lines are supported by the National Institutes of Health, National Center for Research Resources, Biomedical Technology Program, and by the Department of Energy, Office of Biological and Environmental Research.

\section{REFERENCES}

1. Hatefi, Y., and Yamaguchi, M. (1996) FASEB J. 10, 444-452.

2. Hatefi, Y., and Yamaguchi, M. (1992) in Molecular Mechanisms in Bioenergetics (Ernster, L., Ed.) pp 265-281, Elsevier Science Publisher, Amsterdam.

3. Jackson, J. B., Quirk, P. G., Cotton, N. P. J., Venning, J. D., Gupta, S., Bizouam, T., Peake, S. J., and Thomas, C. M. (1998) Biochim. Biophys. Acta 1365, 79-86.
4. Rydström, J., Hu, X., Fjellström, Meuller, J., Zhang, J., Johansson, C., and Bizouam, T. (1998) Biochim. Biophys. Acta 1365, 10-16.

5. Yamaguchi, M., and Hatefi, Y. (1993) J. Biol. Chem. 268, 1787117877.

6. Yamaguchi, M., and Hatefi, Y. (1997) Biochim. Biophys. Acta $1318,225-234$.

7. Prasad, G. S., Sridhar, V., Yamaguchi, M., Hatefi, Y., and Stout, C. D. (1999) Nat. Struct. Biol. 6, 1126-1131.

8. White, S. A., Peake, S. J., McSweeney, S., Leonard, G., Cotton, N. P. J., and Jackson, J. B. (2000) Structure 8, 1-12.

9. Buckley, P. A., Jackson, J. B., Schneider, T., White, S. A., Rice, D. W., and Baker, P. J. (2000) Structure 8, 809-815.

10. Cotton, N. P. J., White, S. A., Peake, S. J., McSweeney, S., and Jackson, J. B. (2001) Structure 9, 165-176.

11. Jackson, J. B., White, S. A., Quirk, P. G., and Venning, J. D. (2002) Biochemistry 41, 4173-4185.

12. Doublié, S. (1997) Methods Enzymol. 276, 523-530.

13. Collaborative Computational Project, No. 4 (1994) Acta Crystallogr. D50, 760-763.

14. Howell, P. L., Blessing, R. H., Smith, G. D., and Weeks, C. M. (2000) Acta Crystallogr. D56, 604-617.

15. Blessing, R. H., and Smith, G. D. (1999) J. Appl. Crystallogr. 32 , 664-670.

16. De La Fortelle, E., and Bricogne, G. (1997) Methods Enzymol. 276, 472-494.

17. Cowtan, K. (1994) Jt. CCP4 ESF-EACBM Newslett. 31, 34-38. 18. McRee, D. E. (1999) J. Struct. Biol. 125, 156-165.

19. Brünger, A. T., Adams, P. D., Clore, G. M., DeLano, W. L., Gros, P., Grosse-Kunstleve, R. W., Jiang, J.-S., Kuszewski, J., Nilges, M., Pannu, N. S., Read, R. J., Rice, L. M., Simonson, T., and Warren, G. L. (1998) Acta Crystallogr. D54, 905-921.

20. Bourgeois, D., Vernede, X., Adam, V., Fioravanti, E., and Ursby, T. (2002) J. Appl. Crystallogr. 35, 319-326.

21. McRee, D. E. (2001) Molecular Images Software, San Diego, CA.

22. Carson, M. (1997) Methods Enzymol. 277, 493-505.

23. Young, L., and Post, C. B. (1996) Biochemistry 35, 15129-15133.

24. Venning, J. D., Peake, S. J., Quirk, P. G., and Jackson, J. B. (2000) J. Biol. Chem. 275, 19490-19497.

25. Venning, J. D., Rodrigues, D. J., Weston, C. J., Cotton, N. P. J., Quirk, P. G., Errington, N., Finet, S., White, S. A., and Jackson, J. B. (2001) J. Biol. Chem. 276, 30678-30685.

26. Yamaguchi, M., and Hatefi, Y. (1995) J. Biol. Chem. 270, 2816528168

27. Deng, K., Aliverti, A., Zanetti, G., Arakak, A. K., Ottado, J., Orellano, E. G., Calcaterra, N. B., Ceccarelli, E. A., Carillo, N., and Karplus, P. A. (1999) Nat. Struct. Biol. 6, 847-853.

28. Barycki, J. J., O'Brien, L. K., Strauss, A. W., and Banaszak, L. J. (2000) J. Biol. Chem. 275, 27186-27196.

29. Read, J. A., Winter, V. J., Eszes, C. M., Sessions, R. B., and Brady, R. L. (2001) Proteins: Struct., Funct., Genet. 43, 175-185.

30. Karplus, P. A., and Schulz, G. E. (1989) J. Mol. Biol. 210, $163-$ 180 .

31. Pinheiro, T. J. T., Venning, J. D., and Jackson, B. J. (2001) J. Biol. Chem. 276, 44757-44761.

32. Yamaguchi, M., Wakabayashi, S., and Hatefi, Y. (1990) Biochemistry 29, 4136-4143.

33. Tong, R. C. W., Glavas, N. A., and Bragg, P. D. (1991) Biochim Biophys. Acta 1080, 19-28.

34. Phelps, D. C., and Hatefi, Y. (1984) Biochemistry 23, 4475-4480.

35. Quirk, P. G., Jeeves, M., Cotton, N. P. J., Smith, J. K., and Jackson, B. J. (1999) FEBS Lett. 446, 127-132.

36. Yamaguchi, M., Stout, C. D., and Hatefi, Y. (2002) J. Biol. Chem. (in press).

BI020251F 